\title{
LOS MAMÍFEROS DEL ESTADO DE MICHOACÁN
}

\author{
Tiberio C. Monterrubio-Rico ${ }^{1}$, Juan Felipe Charre \\ Medellín ${ }^{1}$, Cristina Z. Colín-Soto ${ }^{1}$, y Livia LeÓN \\ PANIAGUA ${ }^{2}$ \\ 'Laboratorio de Vertebrados Terrestres Prioritarios, Facultad \\ de Biología, Universidad Michoacana de San Nicolás de \\ Hidalgo, Morelia, Michoacán, México C. P. 58194. \\ ${ }^{2}$ Museo de Zoología, Alfonso L. Herrera, Facultad de Ciencias, \\ Universidad Nacional Autónoma de México, México, \\ D. F., México, A. P. 70-153. \\ Autor de correspondencia: Tiberio Monterrubio: \\ tmonter2002@yahoo.com.mx
}

\section{RESUMEN}

Los mamíferos silvestres han sido estudiados en Michoacán desde el siglo XVIII. Sin embargo, la información disponible para el estado se encontraba dispersa. Por lo tanto el objetivo es este análisis es proporcionar una síntesis actualizada sobre la riqueza taxonómica de los mamíferos silvestres terrestres en el estado de Michoacán. Existen registros de 161 especies de 9 órdenes, 25 familias y 94 géneros, que representan el $32 \%$ de las especies de México. El orden con mayor número de especies es Chiroptera con 74 especies que representan el $53 \%$ de las especies del orden en México. A pesar de la ubicación central de Michoacán en el país, y ser un estado muestreado por numerosos investigadores, todavía existe carencia de información para regiones remotas, y en algunas especies. Por ejemplo, del jaguar se carecía de información hasta 2010, a pesar de ser el mayor felino del país. Otro aspecto limitante para la interpretación adecuada de los mamíferos del estado es la antigüedad de muchos registros, ya que los estudios más completos realizados a escala regional, por lo que se desconoce la situación actual que presentan las poblaciones de muchas especies. En Michoacán encuentran su límite más norteño en el Pacífico de tres especies de amplia distribución en el Neotrópico, Tamandua mexicana, Potos flavus y Sphiggurus mexicanus, por lo que deben delimitarse áreas que protejan sus poblaciones.

Palabras clave: Distribución, especies, mamíferos, Michoacán.

\begin{abstract}
The Wild mammals have been studied in Michoacán since the eighteenth century. However, the information available to the state was scattered. Therefore the goal is to this analysis is to provide an update on the taxonomic richness of terrestrial wild mam-
\end{abstract}


mals in the state of Michoacán synthesis. There are records of 161 species of 9 orders, 25 families and 94 genera, representing 32\% of the species in Mexico. The order with the highest number of species is Chiroptera with 74 species representing $53 \%$ of the species of order in Mexico. Despite the central location of Michoacán in the country, and being a sampled by numerous researchers state, there is still lack of information to remote regions, and in some species. For example, the jaguar is no information to 2010 , despite being the largest feline in the country. Another limitation to the proper interpretation of mammals state aspect is the age of many records as the most complete studies at regional level, so that the current situation presented populations of many species are unknown. In Michoacán found its northern boundary in the Pacific three widespread species in the Neotropics, Tamandua mexicana, Potos flavus and Mexican hairy dwarf porcupine, which must be delimited areas to protect their populations.

Key words: Distribution, species, mammals, Michoacan.

\section{INTRODUCCIÓN}

Los mamíferos silvestres en Michoacán han sido estudiados por diversos investigadores desde el siglo XVIII. Renombrados zoólogos y naturalistas han colectado y estudiado a los mamíferos de México en Michoacán. Destacan entre los trabajos más antiguos el de Allen (1895), quien reportó nuevas especies de roedores, o el de Goldman (1918) quien describió las ratas del género Oryzomys. Posteriormente, a mediados del siglo XIX, se incrementaron los estudios de una manera vertiginosa. Hall $(1948,1949)$ documenta nuevos registros para roedores, y Hall y Villa (1948a; $1949 b)$ proporcionan nuevos registros de roedores y de una tuza. Además publican la primer lista de mamíferos tanto en Inglés (Hall y Villa, 1949a), como en Español, (Hall y Villa, 1950). Baker y Alcorn (1953) publicaron el primer estudio sobre musarañas con egagrópilas para Michoacán. Hooper (1957) documentó registros de Reithrodontomys mexicanus para el estado. Cuatro años después Burt (1961) analizó los efectos del volcán Paricutín sobre los vertebrados. Posteriormente Winkelmann en 1962 publicó nuevos registros para Guerrero y Michoacán. Álvarez y Aviña (1965) reportaron los primeros registros de Rhogeessa tumida y R. párvula para Michoacán. Álvarez (1968) publicó los registros existentes en una colección de ejemplares provenientes de la zona costera del Balsas entre Michoacán y Guerrero. Genoways (1973) publicó un estudio sobre la sistemática de los ratones del Género Liomys incluyendo ejemplares colectados en Michoacán. Por su parte Carleton (1977) analizó las relaciones de poblaciones de Peromyscus boylli. Posteriormente se publicaron varios estudios sobre comunidades de mamíferos y de vertebrados, entre los que destacaron Uribe-Peña et al. (1981), quienes publicaron un listado sobre vertebrados del rancho "El Reparito" del Municipio de Arteaga. Otros autores al siguiente año analizaron la variación morfométrica de Peromyscus alstoni (Williams y Ramírez-Pulido, 1984). Otro estudio fue el de SánchezHernández et al. (1985), quienes analizaron la distribución y reproducción de Quirópteros en la costa de Michoacán. Polaco y Muñiz-Martínez (1987) proporcionaron el primer listado compre- 
hensivo de los Quirópteros de la costa Michoacana. En el mismo año, Álvarez y colaboradores (1987) proporcionaron un listado de mamíferos terrestres no voladores para la costa de Michoacán. En la década de los noventas se incrementaron los estudios que proporcionaron nuevos registros y faunas locales. $A$ una escala de ecoregión se analizaron los mamíferos y las áreas protegidas del cinturón Eje Neovolcánico (Fa y Morales, 1991). El siguiente año se analiza la distribución de Nelsonia neotomodon (Glendinning, 1992), y Sánchez y colaboradores (1992) reportaron por primera vez registros de Tamandua mexicana para la costa de Michoacán. Álvarez y Sánchez-Casas (1997) analizaron los mamíferos del estado de talla mediana y grande del estado. En 1998, Uribe y Arita proporcionaron un panorama de la diversidad y distribución de los mamíferos de importancia cinegética en México, incluyendo Michoacán. Sánchez et al. (1999) proporcionaron un listado de registros novedosos para Michoacán, mientras que Orduña y sus colaboradores (2000) publicaron un listado de los mamíferos del altiplano Tarasco. También algunos estudios en sistemática, como el de Thomomys umbrinus (Castro-Campillo y RamirezPullido, 2000).

Durante la última década se aportaron nuevos registros de especies de las que casi no existía información, y para otras especies se confirmó su presencia más allá de los registros de tipo anecdóticos, como el estudio local de los mamíferos del parque nacional "Barranca del Cupatitzio" donde reportaron la presencia de Leopardus wiedii (Chávez-León y Zaragoza, 2009). Entre los últimos estudios más relevantes a nivel estatal destacaron los de Monterrubio-Rico et al. 2010, quienes reportan nuevos registros de distribución de Sphiggurus mexicanus delimitando a la población más norteña de la especie en el continente por el Pacífico; en otro estudio, se presentaron nuevos registros de distribución de Herpailurus yagouaroundi para Michoacán, y se proporcionan sus primeras secuencias del gen de citocromo $b$ para la especie en el trópico seco (Monterrubio-Rico et al., 2012). Charre-Medellín et al. (2010), registraron con cámaras trampa la actividad de vertebrados utilizando manantiales en la región de la costa de Michoacán confirmando para la costa la presencia de tres felinos en riesgo. Charre-Medellín (2012) proporcionaron un listado de 19 especies de mamíferos muestreados mediante trampas cámara en donde se destaca múltiples registros de cuatro especies en peligro de extinción (Panthera onca, Leopardus pardalis, Leopardus wiedii y Tamandua mexicana), y dos amenazadas (Herpailurus yagouaroundi y Spilogale pygmaea), destacándose los primeros registros poblacionales de jaguar para el estado de Michoacán.

Como resultado del incremento de estudios regionales y locales en los últimos 70 años, además de cambios taxonómicos que modificaron la cifra total de mamíferos de México, la estimación sobre la riqueza de especies en Michoacán ha cambiado. Para la primer mitad del siglo XX se reportaban 85 especies (Hall y Villa, 1949a), posteriormente Hall (1981) reportó 141; Ramírez-Pulido et al. (1986) estableció la riqueza estatal en 116; Núñez (2005) reportó 161 especies incluyendo roedores introducidos como Mus musculus, Rattus norvegicus, Rattus rattus, siendo en realidad la cifra de especies silvestres de 158. En ese mismo año en el estudio estado CONABIO (2005) reportó en sus bases de datos de registros de 146 especies. 


\section{SITIO DE ESTUDIO}

El estado de Michoacán fue creado el 31 de enero de 1824. Su nombre deriva de la palabra Náhuatl Michoacán que etimológicamente significa "lugar de los que poseen el pescado." En épocas prehispánicas formó parte del estado Purepecha. Durante la Colonia, Michoacán conformaba junto con Nayarit, Aguascalientes, Colima, Jalisco y Guanajuato de la Provincia de la Nueva Galicia. El estado está situado en la región centro-occidente de la República Mexicana, se localiza entre las coordenadas $20^{\circ} 24^{\prime}$ y $17^{\circ} 55^{\prime}$ de latitud Norte; y los $100^{\circ} 04^{\prime}$ y $103^{\circ}$ de longitud Oeste. Abarca una superficie de $59,864 \mathrm{~km}^{2}$ equivalente al $3 \%$ de la extensión territorial del país y se divide en $113 \mathrm{mu}$ nicipios (INEGI, 2007). Para el estado se reconocen cinco regiones fisiográficas (CONABIO, 2005).

La Planicie Costera del Pacífico con una estrecha franja entre el océano Pacífico y la Sierra Madre del Sur y que es la región de menor extensión con $782 \mathrm{~km}^{2}$ y una amplitud media de $3 \mathrm{~km}$ y las alturas máximas son de $60 \mathrm{msnm}$ (Bocco et al., 1999; Correa, 2003). La Sierra Madre del Sur, se extiende unos $200 \mathrm{~km}$ a lo largo del estado y cubre una superficie aproximada de 13000 $\mathrm{km}^{2}$, con una anchura de casi $100 \mathrm{~km}$ y una altitud promedio de 2000 metros. Presenta grietas y fallas al ser una zona de gran actividad tectónica (CONABIO, 2007). La Depresión del Balsas, tiene una superficie aproximada de 14,000 $\mathrm{km}^{2}$ y presenta una característica fisiográfica particular, ya que es la única depresión tropical interior del país, donde los cambios de altitud son notables propiciando la formación de cañadas y cambios en la cobertura vegetal (Bocco et al., 1999; SEMARNAT y CONAGUA, 2007). Al norte de esta depresión se localiza el Sistema Volcánico Transversal, for- mado como consecuencia de una gran actividad volcánica, este sistema cubre una superficie de $27,500 \mathrm{~km}^{2}$, con al menos 50 volcanes que superan los 2,700 metros de altitud; destacando el Pico de Tancítaro, Patamban, y Nahuatzen. En esta zona también se presentan numerosos valles y cuencas donde se localizan los principales lagos del estado Pátzcuaro, Zirahuén y Cuitzeo. Por último, la Depresión del Lerma, que cubre una extensión de $4,100 \mathrm{~km}^{2}$ y está limitada al sur por el Sistema Volcánico Transversal, se encuentra conformada por grandes planicies separadas por algunas eminencias situadas a distintas altitudes (CONABIO, 2007).

Los climas predominantes en el estado son los tropicales con presencia en el $54.5 \%$ del estado, se localizan en las Planicies costeras y Sierra Madre del Sur, el 29.0\% del territorio es de clima templado en el Sistema Volcánico Transversal y porciones elevadas de la Sierra Madre del Sur. El 15\% constituye cálidos-secos en las partes bajas y medias de la depresión del Balsas y Tepelcatepec, el $1 \%$ del territorio es de clima templado húmedo, y el $0.5 \%$ cálido húmedo se presentan en regiones altas de Sistema Volcánico Transversal (Bocco et al., 1999; García, 1990; INEGI, 2007). La variedad climática, la diversidad fisiográfica y litológica han permitido el desarrollo de una diversidad de tipos de suelo con litosoles, rendzinas, andosoles, regosoles, vertisoles y fluvisoles. Michoacán cuenta con siete sistemas fluviales, la Cuenca del río Lerma, la Cuenca del Balsas Tepalcatepec, y el sistema fluvial costero, el Lago de Cuitzeo, el Lago de Pátzcuaro, el Lago de Zirahuén y el Lago de Chapala (Bocco et al., 1999). Las diversas condiciones ecológicas en el estado reflejan la presencia de numerosas comunidades vegetales, tales como bosque de Abies, bosque mesófilo de montaña, bosque 
de pino, bosque de encino, bosque de junípero, pastizales, matorral xerófilo y vegetación acuática y subacuática (Rzedowski, 1981). Michoacán es uno de los estados con mayor riqueza de flora y fauna del país, ocupa el quinto lugar entre las entidades biológicamente más ricas del país (ConABIO, 2005). Esta elevada diversidad es resultado de la diversidad de climas, vegetación, historia geológica y aislamiento ecológico de comunidades causado por barreras físico-ecológicas propiciando que a diferentes latitudes y altitudes se presenten mosaicos de condiciones ambientales y microambientales. En las zonas de contacto entre las 5 regiones fisiográficas del estado encontramos mezclados ambientes áridos, tropicales, y templado-fríos y esto se ve reflejado en la alta riqueza de especies de vertebrados estimada para el estado (CONABIO, 2005).

De acuerdo al censo realizado por INEGI en 2010, la población humana tuvo un aumento de casi $9 \%$ en cinco años (2005-2010). La mujer representa el $51.6 \%$ de la población estimada en $4,351,037$ que representa el $4 \%$ de la población total del país (INEGI, 2010). En el estado habitan aproximadamente 122,000 habitantes de lengua indígena, concentrados principalmente en los municipios del centro-norte, la costa, y el oriente del estado. En Michoacán se hablan al menos 46 dialectos indígenas, predominando el Purépecha $(117,221$ habitantes), náhuatl $(9,170$ habitantes), mazahua (5,431 habitantes), mixtecos (1,160 habitantes) y otomíes (592 habitantes), quienes en conjunto son el $95 \%$ de los hablantes de lengua indígena (INEGI, 2004; INEGI, 2010). El 27.2\% de la población total se encuentra entre los 15-29 años. La distribución geográfica de la población no es uniforme, ya que cada vez muestra mayor tendencia a concentrarse en las principales loca- lidades urbanas. El $21 \%$ en localidades de entre 2,500 y 15 mil habitantes, el $22.4 \%$ en asentamientos entre 15 mil y 100 mil habitantes, y el $24.6 \%$ se concentra en las tres ciudades de más de 100 mil personas, Morelia, Uruapan, y Zamora (CONABIO, 2007). Las actividades primarias representan el $11.2 \%$ de aportación al PIB estatal, mientras que las actividades secundarias el $19.9 \%$, y a las actividades terciarias el $68.7 \%$ (INEGI, 2011).

\section{MÉTODOS}

La investigación documental reveló que el conocimiento de los mamíferos para el estado es fragmentado y disperso, por lo que se revisó la información presente en publicaciones tanto históricas como artículos de investigación publicados y tesis de licenciatura y posgrado desarrolladas recientemente, las cuales no han sido publicadas todavía. Además de la revisión de literatura, se revisó las bases de datos de que dispone conABIO con registros de mamíferos para Michoacán. La base de datos incluye 9,186 registros (principalmente Rodentia y Quiroptera) provenientes de 10 distintos proyectos de investigación apoyados por conABIO destacando por el número de registros los proyectos "P020 Biodiversidad de los Mamíferos de Michoacán," correspondientes al periodo de colecta de 1967 a 1978 con 6537 registros (Álvarez y López, 1998), el proyecto "T009 Actualización de la base de datos del Atlas Mastozoológico de México con 1269 registros (Ceballos, 2002), y el proyecto "P130 Base de datos de mamíferos de México depositados en colecciones de Estados Unidos y Canadá" (LópezWilchis, 1998) con 954 registros. Además se revisó las bases de datos de proyectos de investigación y de tesis de licenciatura y posgrado efectuados 
en el Laboratorio de Vertebrados Terrestres Prioritarios de la Facultad de Biología de la Universidad Michoacana de San Nicolás de Hidalgo (aproximadamente 11,543 registros) que incluyen registros de una tesis de maestría con 5,963 registros de mamíferos medianos y grandes (Charre-Medellín, 2012). Además de los registros de varias tesis de licenciatura que incluye inventarios obtenidos en campo para los hábitats terrestres de Cuitzeo con 580 registros (Guido-Lemus, 2012), la sub-cuenca del Cupatitzio con 353 registros de Quirópteros (Castro-Vázquez, 2012), las regiones Costa, Sierra, Balsas y Municipio de los Reyes con 568 registros de Quirópteros (Colín-Soto, 2012), así como 134 registros de Roedores para las mismas regiones (Mejía-Ángeles, 2012). Se revisó además para musarañas a Carraway (2007) y los registros obtenidos en los proyectos Fondo Mixto CONACYT-Gobierno del Estado de Michoacán, "Diagnóstico y propuestas sobre el potencial de manejo de la fauna silvestre en el bajo balsas." Clave 41168, y del proyecto de Fondo Mixto CONACYTGobierno del Estado de Michoacán titulado "Saneamiento, Restauración y Conservación de la Subcuenca del Río Cupatitzio", aprobado por el fideicomiso de Fondos Mixtos del Estado de Michoacán-CONACYT (FOMYX-Michoacán), con clave: 115897 efectuado en el año 2010. El listado final incluyó el cruce de información de las distintas fuentes con la relación de mamíferos de México proporcionada para este capítulo.

\section{RESULTADOS}

\section{Diversidad de especies}

Existe evidencia de que en el estado de Michoacán pueden estar presentes 161 especies de mamíferos silvestres terrestres que representan el
$32 \%$ de las especies a nivel nacional. Esta riqueza se encuentra agrupada en 9 Ordenes, 25 familias, y 94 géneros. El Orden con mayor representación de especies es el Chiroptera con 74 especies que representan el $53 \%$ de las especies de murciélagos de México. El segundo es Rodentia con 52 especies que representan el $21.2 \%$ de las especies de roedores de México, y en tercer lugar tenemos el Orden Carnívora con 18 especies que representan el $52 \%$ de las especies de carnívoros del País (Cuadro 1).

\section{Endemismos}

En Michoacán se encuentran 41 especies endémicas de México, destacando el Orden Rodentia con 23 especies endémicas de México y dos especies endémicas estatales (Peromyscus sagax y Zygogeomys trichopus). En el Orden Chiroptera se presentan nueve especies endémicas a México y una especie endémica estatal (Rhogeessa mira).

\section{Conservación}

En el estado se presentan poblaciones importantes de mamíferos listados en categorías de riesgo para el país, incluyendo seis especies en peligro de extinción como Tamandua mexicana, Musonycteris harrisoni, Panthera onca, Leopardus pardalis, Leopardus wiedii y Zygogeomys trichopus, confirmando la presencia actual de poblaciones para cinco de las especies, exceptuando para la tuza Zygogeomys trichopus (Charre-Medellín, 2012; Colín-Soto, 2012). En el estado también hay poblaciones de 11 especies listadas en categoría de Amenazadas, destacando que para las especies Choeronycteris mexicana, Herpailurus yagouaroundi, Lontra longicaudis, Spilogale pygmaea, y 
Sphiggurus mexicanus hemos generado nuevos registros para distintas regiones del estado (Monterrubio-Rico et al., 2010; Monterrubio-Rico et al., 2012; Charre-Medellín, 2012; Guido-Lemus, 2012). Once especies se encuentran listadas en la categoría de Protección especial, de las cuales destaca Potos flavus, especie cuyos nuevos registros en Michoacán establecen su límite más norteño por el Pacífico, y para el trópico seco. Su presencia en Michoacán se consideraba poco probable ya que los pocos registros eran de tipo anecdóticos (Sánchez-Hernández y Gaviño de la Torre, 1988). Sin embargo durante los últimos años se obtuvo registros que confirmaron su presencia (Figura 1). Otra especie notable es la ardilla endémica Sciurus oculatus (Figura 2), la cual no aparecía en los listados de especies con presencia confirmada para Michoacán en documentos anteriores (CONABIO, 2005), a pesar de existir tres localidades con registros históricos de su presencia. Durante los últimos años se obtuvo registros de una población que se desconocía en bosques de encino del municipio de Huandacareo en la cuenca del lago de Cuitzeo.

\section{DISCUSIÓN}

A pesar de estar Michoacán ubicado en el centro del país, y de que es un estado al que numerosos investigadores y naturalistas han realizado estudios sobre su fauna, todavía existe carencia de información para diversas regiones y para numerosas especies. Un ejemplo claro lo es el caso del jaguar. A pesar de ser el felino con mayor tamaño del país, se carecía de datos que confirmaran su presencia para el estado. Por fortuna durante el año 2010, se obtuvo el primer conjunto de registros de la especie para el estado, tanto registros fotográficos (Figura 3), como un cráneo que se depositó en la colección de Mamíferos del Museo de Zoología de la Facultad de Ciencias.

Todavía existen municipios y localidades para las que se carece de estudios sobre los patrones ecológicos locales, estructuras regionales y locales de las comunidades de mamíferos. Otro aspecto limitante es la carencia de datos recientes (últimos 10 años) para regiones enteras de Michoacán. Por ejemplo, el estudio más completo realizado en la costa Michoacana fue hace

Cuadro 1. Composición sistemática de los mamíferos de Michoacán, México.

\begin{tabular}{|l|c|c|c|c|}
\hline ORDEN & FAMILIAS & GÉNEROS & ESPECIES & $\begin{array}{l}\text { ESPECIES } \\
\text { ENDÉMICAS }\end{array}$ \\
\hline DIDELPHIMORPHIA & 1 & 2 & 2 & 1 \\
\hline CINGULATA & 1 & 1 & 1 & 0 \\
\hline PILOSA & 1 & 1 & 1 & 0 \\
\hline SORICOMORPHA & 1 & 4 & 74 & 9 \\
\hline CHIROPTERA & 8 & 40 & 18 & 1 \\
\hline CARNIVORA & 5 & 16 & 2 & 0 \\
\hline ARTIODACTYLA & 2 & 2 & 52 & 23 \\
\hline RODENTIA & 5 & 26 & 3 & 1 \\
\hline LAGOMORPHA & 1 & 2 & 161 & 41 \\
\hline TOTAL & 25 & 94 & & 9 \\
\hline
\end{tabular}




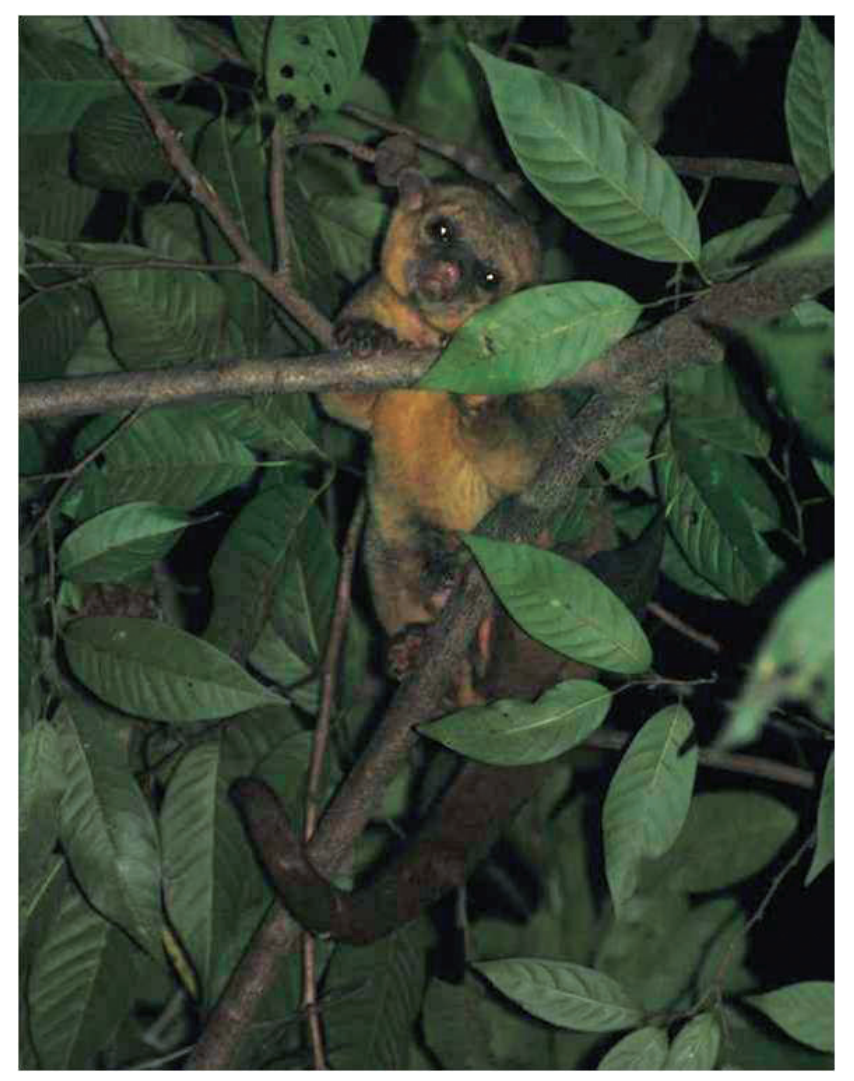

Figura 1. Registro de Potos flavus en Michoacán.

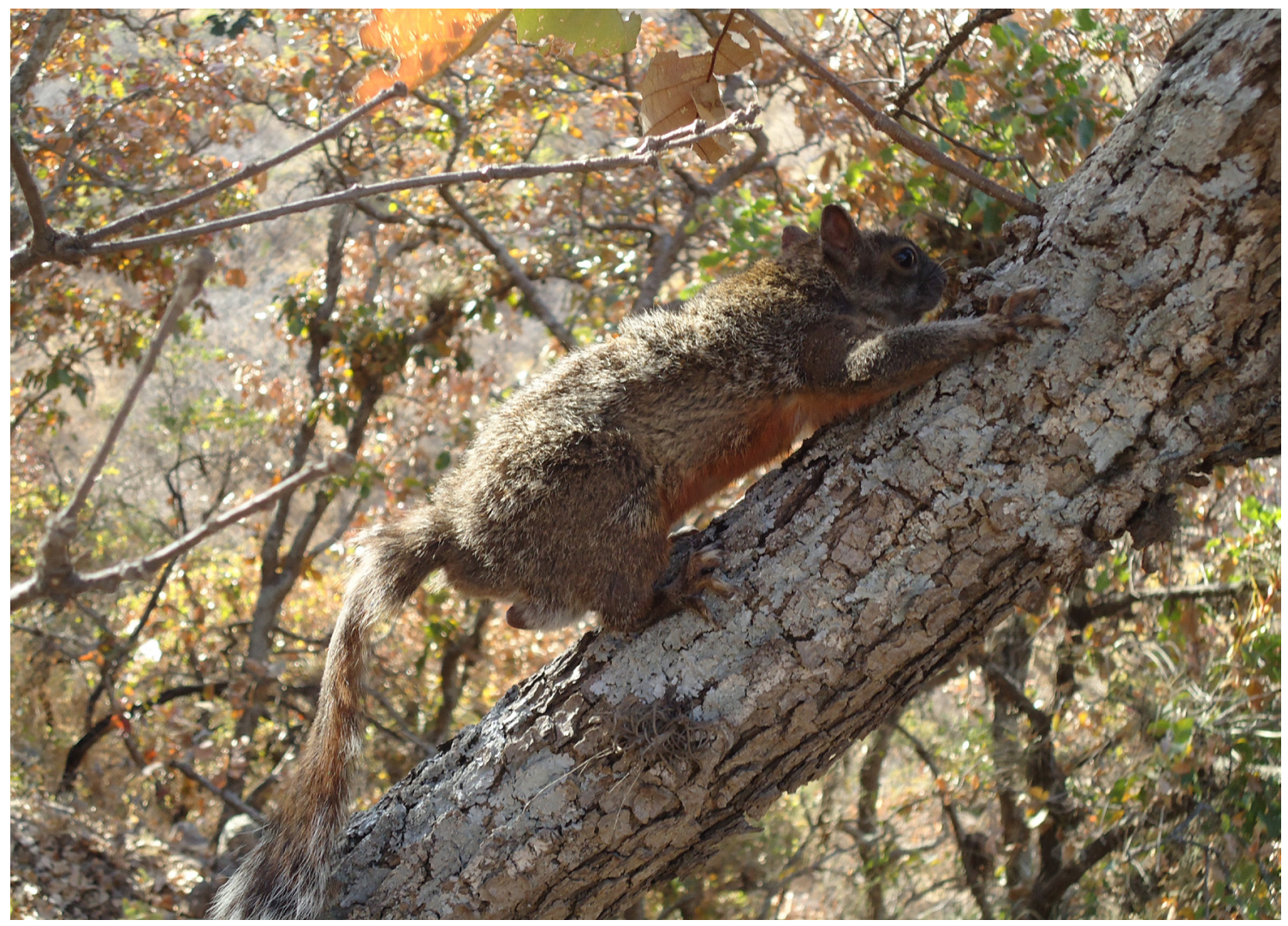

Figura 2. Registro de Sciurus occulatus en Michoacán. 


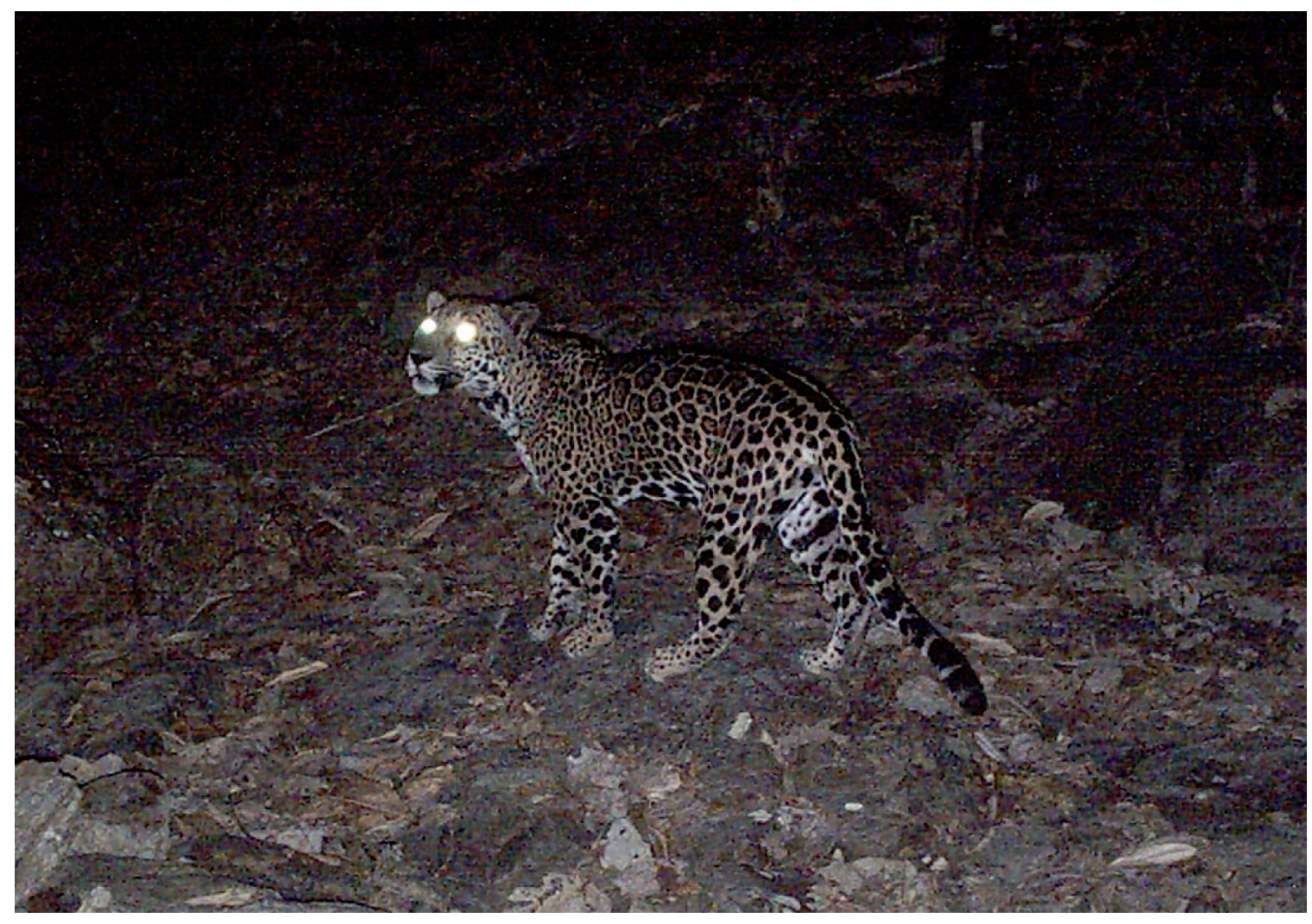

Figura 3. Registro de Panthera onca en Michoacán.

30 años por Polaco y Muñiz (1987), y durante los últimos 10 años se han incrementado las tasas de deforestación, especialmente en las selvas tropicales, por lo que desconocemos la situación actual que presentan las poblaciones de mamíferos de la región de la costa del Pacífico en Michoacán. Ante este panorama, es necesario actualizar la información sobre la situación que presentan las comunidades de Mamíferos en el estado, especialmente en regiones como la Costa, la Sierra Madre del Sur, y la depresión del Balsas. Otra región con pocos registros es el límite entre Michoacán y Jalisco en el altiplano, región que presenta distintos tipos de vegetación como el matorral tropical y el bosque de encino para los que desconocemos su composición mastofaunistica.

\section{LITERATURA CITADA}

Allen, J.A. 1895. On the species of the genus Reithrodontomys. Bulletin of the American Museum of Natural History, 7:107-143.

Álvarez, T. 1968. Notas sobre una colección de mamíferos de la región costera del Río Balsas entre Michoacán y Guerrero. Revista de la Sociedad Mexicana de Historia Natural, 29:21-35.

Álvarez, T., J. Arroyo-Cabrales y M. González-Escamilla. 1987. Mamíferos (excepto Chiroptera de la costa de Michoacán, México). Anales de la Escuela Nacional de Ciencias Biológicas, 31: 13-62.

Álvarez, T. y C.E. Aviña. 1965. Baeodon alleni, Rhogeessa tumida major and R. p. párvula newly reported for Michoacan with notes on the qualitative differentiation of the two Rhogeessas. Southwestern Naturalist, 10:75-76.

Álvarez, T. y N. Sánchez-Casas. 1997. Contribución al conocimiento de los mamíferos, excepto Chiroptera y Rodentia, de Michoacán, México. Anales de la Escuela Nacional de Ciencias Biológicas, 42:47-74.

Álvarez-Solórzano, T. y J.C. López-Vidal. 1998. Biodiversidad de los mamíferos en el Estado de Michoacán. Instituto Politécnico Nacional. Escuela Nacional de Ciencias Biológicas. Base de datos SNIB2010-conABIO 
proyecto No. P020. México D.F.

Baker, R.H. y A. A. Alcorn. 1953. Shrews from Michoacán, México, found in barn owl pellets. Journal of Mammalogy, 34:116.

Bocco G., M.E. Mendoza, A. Velázquez y A. Torres. 1999. La regionalización geomorfológica como una alternativa ecológica en México, el caso de Michoacán de Ocampo. Investigaciones Geográficas, Boletín 40.

Burt, W.H. 1961. Some effects of volcan Paricutin on vertebrates. Occasional Papers of the Museum of Zoology, University of Michigan, 620:1-24.

Carleton, M.D. 1977. Interrelationships of populations of the Peromycus boylli species group (Rodentia, Muridae) in western Mexico. Occasional papers of the Museum of Zoology, University of Michigan, 675:1-47.

Carraway, L.N. 2007. Shrews (Eulypotyphla : Soricidae) of Mexico. Monograph of the Western North American Naturalist, Monograph No. 3:1-91.

Castro-Campillo, A. y J. RamírezPulido. 2000. Systematics of the smooth-toothed gopher, Thomomys umbrinus, in the Mexican Transvolcanic Belt. American Museum Novitates, 329:1-37.

Castro-Vázquez, R. 2012. Quirópteros de la Subcuenca del Río Cupatitzio, Michoacán, México. Tesis de Licenciatura. Facultad de Biología. Universidad Michoacana de San Nicolás de Hidalgo.

Ceballos, G. 2002. Actualización de la base de datos del Atlas Mastozoologico de México. Universidad Nacional Autónoma de México. Instituto de Ecología. Base de datos SNIB2010CONABIO proyecto No. T009. México D. F.

Charre-Medellín, J.F., Colín-Soto, C.Z. y T.C. Monterrubio-Rico. 2010. Uso de manantiales de filtración por los vertebrados durante la época seca en un bosque tropical fragmentado en la costa de Michoacán. Acta Zoológica Mexicana (nueva serie), 26(3):737-743.

Charre-Medellín, J.F. 2012. Uso de manantiales por los mamíferos silvestres en bosques tropicales de Michoacán. Tesis de grado de Maestro en Ciencias en Conservación y Manejo de Recursos Naturales. Facultad de Biología, Maestría
Institucional en Ciencias Biológicas. Universidad Michoacana de San Nicolás de Hidalgo.

Chávez-León, G. y S.R. Zaragoza. 2009. Riqueza de mamíferos del Parque Nacional Barranca del $\mathrm{Cu}$ patitzio, Michoacán, México. Revista Mexicana de Biodiversidad, 80: 95104.

conabio (Comisión Nacional para el Conocimiento y Uso de la Biodiversidad). 2005. La Biodiversidad en Michoacán, Estudio de Estado. Consejo Nacional para el Conocimiento y Uso de la Biodiversidad, México, D.F. conabio (Comisión Nacional para el Conocimiento y Uso de la Biodiversidad). 2007. Estrategia para la Conservación y Uso Sustentable de la Diversidad Biológica de Michoacán. México.

Colín-Soto, C.Z. 2012. Riqueza y estructura de comunidades del Orden Quiróptera en bosques tropicales de Michoacán. Tesis de Licenciatura. Facultad de Biología Universidad Michoacana de San Nicolás de Hidalgo.

Correa P.G. 2003. Atlas Geográfico del Estado de Michoacán. EDDISA, Morelia.

Fa, J.E. y L.M. Morales. 1991. Mammals and protected areas in the Trans-Mexican Neovolcanic Belt.Pp: 199-226, en: Latin American mammalogy: history, biodiversity, and conservation (Mares M.A. y D.J. Schmidly, eds.). University of Oklahoma Press, Norman, Oklahoma.

García, E. 1981. Modificación climática de Köppen. Universidad Nacional Autónoma de México, México, D.F.

Genoways, H.H. 1973. Systematics and evolutionary relationships of spiny pocket mice, genus Liomys. Special Publications the Museum, Texas Tech University, 5:1-368.

Glendinning, J.I. 1992. Range extension for the diminutive woodrat, Nelsonia neotomodon, in the Mexican Transvolcanic range. Southwestern Naturalist, 37: 92-93.

Goldman, E.A. 1918. The rice rats of North America (Genus Oryzomys). North American Fauna, 43:1-100.

Guido-Lemus, D. 2012. Riqueza de la comunidad de los mamíferos silvestres de la cuenca del Lago de Cuitzeo, Michoacán, una comparación utilizando métodos de muestreo. Tesis de Licenciatura. Facultad de
Biología, Universidad Michoacana de San Nicolás de Hidalgo.

Hall, E.R. 1948. Two new meadow mice from Michoacan, Mexico. University of Kansas Publications, Museum of Natural History, 1: 423-427.

Hall, E.R. 1949.A new subspecies of cotton rat, Sigmodon hispidus, from Michoacan, Mexico. Proceedings of the Biological Society of Washington, 62: 149-150.

Hall, E.R. 1981. The mammals of North America. John Wiley and Sons, vol. 1: XV+600+90, vol. 2: VI+601$1181+90$.

Hall, E.R. y B. Villa R. 1948. A new pocket gopher (Thomomys) and a new spiny pocket mouse (Liomys) from Michoacan, Mexico. University of Kansas Publications, Museum of Natural History, 1: 249-256.

Hall, E.R. y B. Villa R. 1949a. An annotated check list of the mammals of Michoacan, Mexico. University of Kansas Publications, Museum of Natural History, 1:431-472.

Hall, E.R. y B. Villa R. 1949b. A new harvest mouse from Michoacan, Mexico. Proceedings of the Biological Society of Washington, 62: 163-164.

Hall, E.R. y B. Villa R. 1950. Lista anotada de los mamíferos de Michoacán, México. Anales del Instituto de Biolog ía, Universidad Nacional Autónoma de México, 21:159-214.

Hooper, E.T. 1957. Record of the Mexican harvest mouse (Reithrodontomys mexicanus) from Michoacan, Mexico. Journal of Mammalogy, 38: 521-522.

INEGI (Instituto Nacional de Estadística y Geografía). 2004. La población hablante de lengua indígena de Michoacán de Ocampo. Dirección General de Coordinación de los Sistemas Nacionales Estadístico y de Información Geográfica. Aguascalientes, Aguascalientes. México.

INEGI (Instituto Nacional de Estadística y Geografía). 2007. Censo Agropecuario: Características del sector agropecuario y forestal en Michoacán. México.

INEGI (Instituto Nacional de Estadística y Geografía). 2010. Censo de Población y Vivienda. México.

INEGI (Instituto Nacional de Estadística y Geografía). 2011. Sistema de Cuentas Nacionales de México. Producto Interno Bruto por entidad federati- 
va. Aguascalientes, Aguascalientes. México.

López-Wilchis, R. 1998. Bases de datos de mamíferos de México depositados en Colecciones de Estados Unidos y Canadá. Universidad Autónoma Metropolitana-Iztapalapa. División de Ciencias Biológicas y de La Salud. Base de datos SNIB2010conabio proyecto No. P130. México D.F.

Mejia-Angeles, C. 2012. Riqueza composición y estructura de comunidades de pequeños roedores en localidades tropicales de Michoacán. Tesis de Licenciatura. Facultad de Biología. Universidad Michoacana de San Nicolás de Hidalgo.

Monterrubio-Rico T.C., J.M. OrtegaRodríguez, R. Cancino-Murillo, N. Mendoza-Cárdenas y A. PérezArteaga. 2010. Distributional and ecological records of the Mexican hairy dwarf porcupine (Sphiggurus mexicanus) from Michoacan, Mexico. The Southwestern Naturalist, 55 (1):143-145.

Monterrubio-Rico, T.C., J.F. CharreMedellín, M.G. Zavala-Páramo, H. Cano-Camacho, P.R. Mario Quetzal, y L. León-Paniagua. 2012. Evidencias fotográfica, biológica y genética de la presencia actual del jaguarundi (Puma yagouaroundi) en Michoacán, México. Revista Mexicana de Biodiversidad, 83:825-833.

Núñez, A. 2005. Los Mamíferos Silvestres de Michoacán. Diversidad, Bio- logía e Importancia. UMSNH. México.

Orduña T.C., C.A. Castro y P.J. Ramírez. 2000. Mammals from the Tarascan Plateau, Michoacán, México. Revista Mexicana de Mastozoología, 4: 53-68.

Polaco, O. J. y R. Muñiz-Martínez. 1987. Los murciélagos de la costa de Michoacán. Anales de la Escuela Nacional de Ciencias Biológicas, México, 31:68-89.

Ramírez-Pulido, J., M.C. Britton, A. Perdomo y A. Castro. 1986. Guía de los mamíferos de México. Referencias hasta 1983. Ediciones de la Universidad Autónoma Metropolitana, Iztapalapa, México, D.F.

Rzedowski, J. 1981. La vegetación de México. Limusa, México, D.F.

Sánchez-Hernández, C., C.B. ChávezTapia, A Núñez-Garduño, E. CebaIlos-Corona, y M.A. Gurrola-Hidalgo. 1985. Notes on distribution and reproduction of bats from coastal regions of Michoacan, Mexico. Journal of Mammalogy, 66:549-553.

Sánchez- Hernández, C. y G. Gaviño de La Torre. 1988. Registros de tres especies de mamíferos para la región central y occidental de México. Anales del Instituto de Biología, Universidad Nacional Autónoma de México, Serie Zoología, 1:477-478.

Sánchez H.C., M.L. Romero A. y A. G. Núñez. 1992. El oso hormiguero Tamandua mexicana en la costa del Estado de Michoacán. Southwestern Naturalist, 37:88-89.
Sánchez-Hernández, C., M.L. Romero-Almaraz, R.D. Owen, A. Núñez-Garduño, y R. López-Wilchis. 1999. Noteworthy records of mammals from Michoacán, México. Southwestern Naturalist, 44:231235.

Semarnat (Secretaria de Medio Ambiente y Recursos Naturales) y conAGUA (Consejo Nacional del Agua). 2007. Organismo de Cuenca Balsas: Situación de los acuíferos en la cuenca del Río Balsas. Guerrero, México.

Uribe Peña, Z., G. Gaviño de la Torre y C. Sánchez Hernández. 1981. Vertebrados del rancho "El Reparito" Municipio de Arteaga, Michoacán, México. Anales del Instituto de Biología, Universidad Nacional Autónoma de México, Serie Zoología, 51:615-646.

Uribe E.J. y H.T. Arita. 1998. Distribución, diversidad y conservación de los mamíferos de importancia cinegética en México. Acta Zoológica Mexicana (nueva serie), 75:45-71.

Williams S.L. y J. Ramírez-Pulido. 1984. Morphometric variation in the volcano mouse Peromyscus (Neotomodon) alstoni (Mammalia: Cricetidae). Annals of Carnegie Museum, 53:163-183.

Winkelmann, J.R. 1962. Mammal records from Guerrero and Michoacan, Mexico. Journal of Mammalogy, 43:108-109. 
Apéndice I. Lista sistemática de los mamíferos de Michoacán, México.

\begin{tabular}{|c|c|c|c|c|c|}
\hline & \multicolumn{2}{|c|}{ Distribución } & \multicolumn{3}{|c|}{ Estado de Conservación } \\
\hline & Ins/Cont & Continente & SEMARNAT & CITES & IUCN \\
\hline \multicolumn{6}{|l|}{ ORDEN DIDELPHIMORPHIA } \\
\hline \multicolumn{6}{|l|}{ FAMILIA DIDELPHIDAE } \\
\hline \multicolumn{6}{|l|}{ SUBFAMILIA DELPHINAE } \\
\hline Didelphis virginiana Kerr, 1792 & IC & AM & & & \\
\hline Tlacuatzin canescens (J.A. Allen, 1893) & IC & MX & & & \\
\hline \multicolumn{6}{|l|}{ ORDEN CINGULATA } \\
\hline \multicolumn{6}{|l|}{ FAMILIA DASYPODIDAE } \\
\hline \multicolumn{6}{|l|}{ SUBFAMILIA DASYPODINAE } \\
\hline Dasypus novemcinctus Linnaeus, 1758 & IC & AM & & & \\
\hline \multicolumn{6}{|l|}{ ORDEN PILOSA } \\
\hline \multicolumn{6}{|l|}{ FAMILIA MYRMECOPHAGIDAE } \\
\hline Tamandua mexicana (Saussure, 1860) & $\mathrm{C}$ & SA & E & & \\
\hline \multicolumn{6}{|l|}{ ORDEN SORICOMORPHA } \\
\hline \multicolumn{6}{|l|}{ FAMILIA SORICIDAE } \\
\hline \multicolumn{6}{|l|}{ SUBFAMILIA SORICINAE } \\
\hline Cryptotis alticola (Merriam, 1895) & $\mathrm{C}$ & MX & MX & $M X$ & MX \\
\hline Cryptotis parva (Say, 1822) & $\mathrm{C}$ & AM & * & & \\
\hline Megasorex gigas (Merriam, 1897) & $\mathrm{C}$ & $\mathrm{MX}$ & A & & \\
\hline Notiosorex evotis (Coues, 1877) & $\mathrm{C}$ & MX & & & \\
\hline Sorex mediopua Carraway, 2007 & $\mathrm{C}$ & $\mathrm{MX}$ & & & \\
\hline Sorex orizabae Merriam 1895 & $\mathrm{C}$ & $M X$ & & & \\
\hline Sorex saussurei Merriam, 1892 & $\mathrm{C}$ & MA & * & & \\
\hline Sorex veraecrucis Jackson, 1925 & $\mathrm{C}$ & $M X$ & & & \\
\hline \multicolumn{6}{|l|}{ ORDEN CHIROPTERA } \\
\hline \multicolumn{6}{|l|}{ FAMILIA EMBALLONURIDAE } \\
\hline \multicolumn{6}{|l|}{ SUBFAMILIA EMBALLONURINAE } \\
\hline Balantiopteryx plicata Peters, 1867 & IC & SA & & & \\
\hline Diclidurus albus Wied-Neuwied, 1820 & $\mathrm{C}$ & SA & & & \\
\hline Peropteryx macrotis (Wagner, 1843) & $\mathrm{C}$ & SA & & & \\
\hline Saccopteryx bilineata (Temminck, 1838) & $\mathrm{C}$ & SA & & & \\
\hline \multicolumn{6}{|l|}{ FAMILIA NOCTILIONIDAE } \\
\hline Noctilio leporinus (Linnaeus, 1758) & $\mathrm{C}$ & SA & & & \\
\hline \multicolumn{6}{|l|}{ FAMILIA MORMOOPIDAE } \\
\hline Mormoops megalophylla (Peters, 1864) & IC & AM & & & \\
\hline Pteronotus davyi Gray, 1838 & IC & SA & & & \\
\hline Pteronotus parnellii (Gray, 1843) & IC & SA & & & \\
\hline Pteronotus personatus (Wagner, 1843) & IC & SA & & & \\
\hline
\end{tabular}


Apéndice I. Continuación...

\begin{tabular}{|c|c|c|c|c|c|}
\hline & \multicolumn{2}{|c|}{ Distribución } & \multicolumn{3}{|c|}{ Estado de Conservación } \\
\hline & Ins/Cont & Continente & SEMARNAT & CITES & IUCN \\
\hline \multicolumn{6}{|l|}{ FAMILIA PHYLLOSTOMIDAE } \\
\hline \multicolumn{6}{|l|}{ SUBFAMILIA MACROTINAE } \\
\hline Macrotus waterhousii Gray, 1843 & IC & MA & & & \\
\hline \multicolumn{6}{|l|}{ SUBFAMILIA MICRONYCTERINAE } \\
\hline Glyphonycteris sylvestris Thomas, 1896 & $\mathrm{C}$ & SA & & & \\
\hline Micronycteris microtis Miller, 1898 & IC & SA & & & \\
\hline \multicolumn{6}{|l|}{ SUBFAMILIA DESMODONTINAE } \\
\hline Desmodus rotundus (È. Geoffroy St.-Hilaire, 1810) & $\mathrm{C}$ & SA & & & \\
\hline \multicolumn{6}{|l|}{ SUBFAMILIA PHYLLOSTOMINAE } \\
\hline \multicolumn{6}{|l|}{ TRIBE PHYLLOSTOMINI } \\
\hline \multicolumn{6}{|l|}{ TRIBE GLOSSOPHAGINI } \\
\hline Anoura geoffroyi Gray, 1838 & $\mathrm{C}$ & SA & & & \\
\hline Choeroniscus godmani (Thomas, 1903) & $\mathrm{C}$ & SA & & & \\
\hline Choeronycteris mexicana Tschudi, 1844 & $\mathrm{C}$ & NA & A & & NT \\
\hline Glossophaga commissarisi Gardner, 1962 & $\mathrm{C}$ & SA & & & \\
\hline Glossophaga leachii (Gray, 1844) & $\mathrm{C}$ & MA & & & \\
\hline Glossophaga morenoi Martínez \& Villa, 1938 & $\mathrm{C}$ & MX & & & \\
\hline Glossophaga soricina (Pallas, 1766) & $\mathrm{C}$ & SA & & & \\
\hline Hylonycteris underwoodi Thomas, 1903 & $\mathrm{C}$ & MA & & & \\
\hline Leptonycteris yerbabuenae Martínez \& Villa, 1941 & IC & AM & A & & VU \\
\hline Leptonycteris nivalis (Saussure, 1860) & $\mathrm{C}$ & NA & A & & EN \\
\hline Musonycteris harrisoni Schaldach \& McLaughlin, 1960 & $\mathrm{C}$ & MX & $\mathrm{P}$ & & VU \\
\hline \multicolumn{6}{|l|}{ TRIBE STENODERMATINI } \\
\hline Artibeus hirsutus Andersen, 1906 & $\mathrm{C}$ & MX & & & \\
\hline Artibeus jamaicensis Leach, 1821 & IC & SA & & & \\
\hline Artibeus lituratus (Olfers, 1818) & IC & SA & & & \\
\hline Carollia subrufa (Hahn, 1905) & $\mathrm{C}$ & MA & & & \\
\hline Centurio senex Gray, 1842 & $\mathrm{C}$ & SA & & & \\
\hline Chiroderma salvini Dobson, 1878 & $\mathrm{C}$ & SA & & & \\
\hline Dermanura azteca (Andersen, 1906) & $\mathrm{C}$ & MA & & & \\
\hline Dermanura phaeotis Miller, 1902 & IC & SA & & & \\
\hline Dermanura tolteca (Saussure, 1860) & C & MA & & & \\
\hline Enchisthenes hartii (Thomas, 1892) & $\mathrm{C}$ & SA & PE & & \\
\hline Sturnira hondurensis Goodwin, 1940 & $\mathrm{C}$ & MA & & & \\
\hline Sturnira lilium (È. Geoffroy St.-Hilaire, 1810) & $\mathrm{C}$ & SA & & & \\
\hline Uroderma magnirostrum Davis, 1968 & $\mathrm{C}$ & SA & & & \\
\hline \multicolumn{6}{|l|}{ FAMILIA NATALIDAE } \\
\hline Natalus mexicanus Miller, 1902 & C & SA & & & \\
\hline
\end{tabular}


Apéndice I. Continuación...

\begin{tabular}{|c|c|c|c|c|c|}
\hline & \multicolumn{2}{|c|}{ Distribución } & \multicolumn{3}{|c|}{ Estado de Conservación } \\
\hline & Ins/Cont & Continente & SEMARNAT & CITES & IUCN \\
\hline \multicolumn{6}{|l|}{ FAMILIA VESPERTILIONIDAE } \\
\hline \multicolumn{6}{|l|}{ SUBFAMILIA MYOTINAE } \\
\hline Myotis auriculus Baker \& Stains, 1955 & C & AM & & & \\
\hline Myotis californicus (Audubon \& Bachman, 1842) & $\mathrm{C}$ & AM & & & \\
\hline Myotis carteri La Val, 1973 & $\mathrm{C}$ & MX & & & \\
\hline Myotis melanorhinus (Merriam, 1890) & $\mathrm{C}$ & NA & & & \\
\hline Myotis fortidens Miller \& Allen, 1928 & C & MA & & & \\
\hline Myotis occultus Hollister, 1909 & $\mathrm{C}$ & NA & & & \\
\hline Myotis thysanodes Miller, 1897 & $\mathrm{C}$ & NA & & & \\
\hline Myotis velifer (J.A. Allen, 1890) & $\mathrm{C}$ & AM & & & \\
\hline Myotis yumanensis $(\mathrm{H}$. Allen, 1864) & $\mathrm{C}$ & NA & & & \\
\hline \multicolumn{6}{|l|}{ SUBFAMILIA VESPERTILIONINAE } \\
\hline Corynorhinus mexicanus G.M. Allen, 1916 & C & MX & & & NT \\
\hline Corynorhinus towsendii (Cooper, 1837) & IC & NA & & & \\
\hline Eptesicus furinalis (d'Orbigny \& Gervais, 1847) & $\mathrm{C}$ & SA & & & \\
\hline Eptesicus fuscus (Palisot de Beauvois, 1796) & $\mathrm{C}$ & AM & & & \\
\hline Idionycteris phyllotis (G.M. Allen, 1916) & $\mathrm{C}$ & NA & & & \\
\hline Lasiurus blossevillii (Lesson \& Garnot, 1826) & IC & AM & & & \\
\hline Lasiurus borealis (Müller, 1776) & C & NA & & & \\
\hline Lasiurus cinereus (Palisot de Beauvois, 1796) & $\mathrm{C}$ & $\mathrm{AM}$ & & & \\
\hline Lasiurus ega (Gervais, 1856) & $\mathrm{C}$ & AM & & & \\
\hline Lasiurus intermedius H. Allen, 1862 & $\mathrm{C}$ & NA & & & \\
\hline Lasiurus xanthinus (Thomas, 1897) & $\mathrm{C}$ & NA & & & \\
\hline Rhogeessa alleni Thomas, 1892 & C & MX & & & \\
\hline Rhogeessa mira La Val, 1973 & $\mathrm{C}$ & MX & PE & & $\mathrm{VU}$ \\
\hline Rhogeessa parvula H. Allen, 1866 & IC & MX & & & \\
\hline \multicolumn{6}{|l|}{ FAMILIA ANTROZOIDAE } \\
\hline Antrozous pallidus (Le Conte, 1856) & IC & NA & & & \\
\hline \multicolumn{6}{|l|}{ FAMILIA MOLOSSIDAE } \\
\hline \multicolumn{6}{|l|}{ SUBFAMILIA MOLOSSINAE } \\
\hline Cynomops mexicanus (Jones \& Genoways, 1967) & $\mathrm{C}$ & MX & PE & & \\
\hline Eumops perotis (Schinz, 1821) & $\mathrm{C}$ & AM & & & \\
\hline Eumops underwoodi Goodwin, 1940 & $\mathrm{C}$ & AM & & & \\
\hline Molossus molossus (Pallas, 1766) & C & SA & & & \\
\hline Molossus rufus E. Geoffroy, 1805 & $\mathrm{C}$ & SA & & & \\
\hline Molossus sinaloae J.A. Allen, 1906 & $\mathrm{C}$ & SA & & & \\
\hline Nyctinomops aurispinosus (Peale, 1848) & $\mathrm{C}$ & SA & & & \\
\hline Nyctinomops femorosaccus (Merriam, 1889) & $\mathrm{C}$ & NA & & & \\
\hline Nyctinomops laticaudatus (È. Geoffroy St.-Hilaire, 1805) & $\mathrm{C}$ & SA & & & \\
\hline
\end{tabular}


Apéndice I. Continuación...

\begin{tabular}{|c|c|c|c|c|c|}
\hline & \multicolumn{2}{|c|}{ Distribución } & \multicolumn{3}{|c|}{ Estado de Conservación } \\
\hline & Ins/Cont & Continente & SEMARNAT & CITES & IUCN \\
\hline Nyctinomops macrotis (Gray, 1839) & $\mathrm{C}$ & AM & & & \\
\hline Promops centralis Thomas, 1915 & C & SA & & & \\
\hline \multicolumn{6}{|l|}{ SUBFAMILIA TADARINAE } \\
\hline Tadarida brasiliensis (È. Geoffroy St.-Hilaire, 1824) & C & AM & & & \\
\hline \multicolumn{6}{|l|}{ ORDEN CARNIVORA } \\
\hline \multicolumn{6}{|l|}{ FAMILIA CANIDAE } \\
\hline Canis latrans Say, 1823 & IC & NA & & & \\
\hline Urocyon cinereoargenteus (Schreber, 1775) & IC & AM & & & \\
\hline \multicolumn{6}{|l|}{ FAMILIA FELIDAE } \\
\hline \multicolumn{6}{|l|}{ SUBFAMILIA FELINAE } \\
\hline Herpailurus yagouaroundi (Lacépède, 1809) & $\mathrm{C}$ & AM & A & I & EN4 \\
\hline Leopardus pardalis (Linnaeus, 1758) & C & AM & $\mathrm{P}$ & I & EN5 \\
\hline Leopardus wiedii (Schinz, 1821) & C & AM & $\mathrm{P}$ & I & NT \\
\hline Lynx rufus (Schreber, 1777) & C & NA & & II & \\
\hline Puma concolor (Linnaeus, 1771) & C & AM & & & \\
\hline \multicolumn{6}{|l|}{ SUBFAMILIA PANTHERINAE } \\
\hline Panthera onca (Linnaeus, 1758) & $\mathrm{C}$ & AM & $\mathrm{P}$ & & NT \\
\hline \multicolumn{6}{|l|}{ FAMILIA MUSTELIDAE } \\
\hline \multicolumn{6}{|l|}{ SUBFAMILIA LUTRINAE } \\
\hline Lontra longicaudis (Olfers, 1818) & C & SA & A & IV & \\
\hline \multicolumn{6}{|l|}{ SUBFAMILIA MUSTELINAE } \\
\hline Mustela frenata Lichtenstein, 1831 & C & AM & & & \\
\hline \multicolumn{6}{|l|}{ FAMILIA MEPHITINAE } \\
\hline Conepatus leuconotus (Lichtenstein, 1832) & C & NA & & & \\
\hline Mephitis macroura Lichtenstein, 1832 & C & AM & & & \\
\hline Spilogale gracilis Merriam, 1890 & C & NA & & & \\
\hline Spilogale pygmaea Thomas, 1898 & C & MX & A & & VU \\
\hline \multicolumn{6}{|l|}{ FAMILIA PROCYONIDAE } \\
\hline \multicolumn{6}{|l|}{ SUBFAMILIA POTOSINAE } \\
\hline Potos flavus (Schreber, 1774) & $\mathrm{C}$ & SA & $\mathrm{P}$ & III & \\
\hline \multicolumn{6}{|l|}{ SUBFAMILIA PROCYONINAE } \\
\hline Bassariscus astutus (Lichtenstein, 1830) & IC & NA & $*$ & & \\
\hline Nasua narica (Linnaeus, 1766) & C & AM & $*$ & III & \\
\hline Procyon lotor (Linnaeus, 1758) & $\mathrm{C}$ & AM & & & \\
\hline \multicolumn{6}{|l|}{ ORDEN ARTIODACTYLA } \\
\hline \multicolumn{6}{|l|}{ FAMILIA CERVIDAE } \\
\hline \multicolumn{6}{|l|}{ SUBFAMILIA ODOCOILEINAE } \\
\hline Odocoileus virginianus (Zimmermann, 1780) & IC & AM & & & \\
\hline
\end{tabular}


Apéndice I. Continuación...

\begin{tabular}{|c|c|c|c|c|c|}
\hline & \multicolumn{2}{|c|}{ Distribución } & \multicolumn{3}{|c|}{ Estado de Conservación } \\
\hline & Ins/Cont & Continente & SEMARNAT & CITES & IUCN \\
\hline \multicolumn{6}{|l|}{ FAMILIA TAYASSUIDAE } \\
\hline Tayassu pecari (Link, 1795) & $\mathrm{C}$ & SA & p & II & NT \\
\hline \multicolumn{6}{|l|}{ ORDEN RODENTIA } \\
\hline \multicolumn{6}{|l|}{ FAMILIA SCIURIDAE } \\
\hline \multicolumn{6}{|l|}{ SUBFAMILIA SCIURINAE } \\
\hline Glaucomys volans (Linnaeus, 1758) & $\mathrm{C}$ & NA & A & & \\
\hline Ictidomys mexicanus (Erxleben, 1777) & $\mathrm{C}$ & NA & & & \\
\hline Notocitellus adocetus (Merriam, 1903) & $\mathrm{C}$ & $M X$ & & & \\
\hline Notocitellus annulatus (Audubon \& Bachman, 1842) & $\mathrm{C}$ & MX & & & \\
\hline Otospermophilus variegatus (Erxleben, 1777) & IC & NA & & & \\
\hline Sciurus aureogaster F. Cuvier, 1829 & C & MA & & & \\
\hline Sciurus oculatus Peters, 1863 & $\mathrm{C}$ & $M X$ & $\mathrm{PE}$ & & \\
\hline \multicolumn{6}{|l|}{ FAMILIA GEOMYIDAE } \\
\hline Cratogeomys fumosus (Merriam, 1892) & $\mathrm{C}$ & $M X$ & A & & \\
\hline Cratogeomys planiceps (Merriam, 1895) & $\mathrm{C}$ & $M X$ & & & \\
\hline Orthogeomys grandis (Thomas, 1893) & $\mathrm{C}$ & MA & & & \\
\hline Pappogeomys bulleri (Thomas, 1892) & $\mathrm{C}$ & $M X$ & & & \\
\hline Thomomys umbrinus (Richardson, 1829) & $\mathrm{C}$ & NA & & & \\
\hline Zygogeomys trichopus Merriam, 1895 & $\mathrm{C}$ & $M X$ & $\mathrm{P}$ & & EN \\
\hline \multicolumn{6}{|l|}{ FAMILIA HETEROMYIDAE } \\
\hline \multicolumn{6}{|l|}{ SUBFAMILIA HETEROMYINAE } \\
\hline Liomys irroratus (Gray, 1868) & $\mathrm{C}$ & NA & & & \\
\hline Liomys pictus (Thomas, 1893) & $\mathrm{C}$ & MA & & & \\
\hline \multicolumn{6}{|l|}{ SUBFAMILIA PEROGNATHINAE } \\
\hline Perognathus flavus Baird, 1855 & $\mathrm{C}$ & NA & & & \\
\hline \multicolumn{6}{|l|}{ FAMILIA CRICETIDAE } \\
\hline \multicolumn{6}{|l|}{ SUBFAMILIA ARVICOLINAE } \\
\hline Microtus mexicanus (Saussure, 1861) & $\mathrm{C}$ & NA & & & \\
\hline \multicolumn{6}{|l|}{ SUBFAMILIA NEOTOMINAE } \\
\hline Baiomys musculus (Merriam, 1892) & $\mathrm{C}$ & MA & & & \\
\hline Baiomys taylori (Thomas, 1887) & $\mathrm{C}$ & NA & & & \\
\hline Hodomys alleni (Merriam, 1892) & $\mathrm{C}$ & MX & & & \\
\hline Nelsonia goldmani Merriam, 1903 & C & MX & $\mathrm{PE}$ & & EN \\
\hline Neotoma albigula Hartley, 1894 & IC & NA & * & & \\
\hline Neotoma mexicana Baird, 1855 & $\mathrm{C}$ & NA & & & \\
\hline Neotomodon alstoni Merriam, 1898 & $\mathrm{C}$ & MX & & & \\
\hline Oligoryzomys fulvescens (Saussure, 1860) & $\mathrm{C}$ & SA & & & \\
\hline Oryzomys couesi (Alston, 1877) & IC & AM & & & \\
\hline Osgoodomys banderanus (J.A. Allen, 1897) & $\mathrm{C}$ & $M X$ & & & \\
\hline
\end{tabular}


Apéndice I. Continuación...

\begin{tabular}{|c|c|c|c|c|c|}
\hline & \multicolumn{2}{|c|}{ Distribución } & \multicolumn{3}{|c|}{ Estado de Conservación } \\
\hline & Ins/Cont & Continente & SEMARNAT & CITES & IUCN \\
\hline Peromyscus aztecus (Saussure, 1860) & $\mathrm{C}$ & MA & & & \\
\hline Peromyscus boylii (Baird, 1855) & IC & NA & $*$ & & \\
\hline Peromyscus difficilis (J.A. Allen, 1891) & $\mathrm{C}$ & MX & & & \\
\hline Peromyscus gratus Merriam, 1898 & $\mathrm{C}$ & NA & & & \\
\hline Peromyscus hylocetes Merriam, 1898 & $\mathrm{C}$ & MX & & & \\
\hline Peromyscus levipes Merriam, 1898 & $\mathrm{C}$ & MX & & & \\
\hline Peromyscus maniculatus (Wagner, 1845) & IC & NA & $*$ & & \\
\hline Peromyscus melanophrys (Coues, 1874) & C & MX & & & \\
\hline Peromyscus melanotis J.A. Allen \& Chapman, 1897 & $\mathrm{C}$ & NA & & & \\
\hline Peromyscus perfulvus Osgood, 1945 & $\mathrm{C}$ & $M X$ & & & \\
\hline Peromyscus sagax Elliot, 1903 & C & $M X$ & & & \\
\hline Peromyscus spicilegus J.A. Allen, 1897 & $\mathrm{C}$ & $M X$ & & & \\
\hline Peromyscus winkelmanni Carleton, 1977 & $\mathrm{C}$ & MX & PE & & EN \\
\hline Reithrodontomys chrysopsis Merriam, 1900 & $\mathrm{C}$ & $M X$ & & & \\
\hline Reithrodontomys fulvescens J.A. Allen, 1894 & $\mathrm{C}$ & NA & & & \\
\hline Reithrodontomys megalotis (Baird, 1858) & $\mathrm{C}$ & NA & & & \\
\hline Reithrodontomys mexicanus (Saussure, 1860) & $\mathrm{C}$ & SA & & & \\
\hline Reithrodontomys microdon Merriam, 1901 & $\mathrm{C}$ & MA & A & & \\
\hline Reithrodontomys sumichrasti (Saussure, 1861) & $\mathrm{C}$ & MA & & & \\
\hline Reithrodontomys zacatecae Merriam, 1901 & $\mathrm{C}$ & MX & & & \\
\hline Sigmodon alleni Bailey, 1902 & $\mathrm{C}$ & MX & & & VU \\
\hline Sigmodon fulviventer J.A. Allen, 1889 & $\mathrm{C}$ & NA & & & \\
\hline Sigmodon hispidus Say \& Ord, 1825 & $\mathrm{C}$ & AM & & & \\
\hline Sigmodon mascotensis J.A. Allen, 1897 & C & $M X$ & & & \\
\hline \multicolumn{6}{|l|}{ FAMILIA ERETHIZONTIDAE } \\
\hline \multicolumn{6}{|l|}{ SUBFAMILIA ERETHIZONTINAE } \\
\hline Sphiggurus mexicanus (Kerr, 1792) & $\mathrm{C}$ & MA & A & III & \\
\hline \multicolumn{6}{|l|}{ ORDEN LAGOMORPHA } \\
\hline \multicolumn{6}{|l|}{ FAMILIA LEPORIDAE } \\
\hline \multicolumn{6}{|l|}{ SUBFAMILIA LEPORINAE } \\
\hline Lepus callotis Wagler, 1830 & $\mathrm{C}$ & NA & & & NT \\
\hline Sylvilagus cunicularius (Waterhouse, 1848) & $\mathrm{C}$ & $M X$ & & & \\
\hline Sylvilagus floridanus (J.A. Allen, 1890) & C & AM & & & \\
\hline
\end{tabular}

